Special Issue of the 6th International Congress \& Exhibition (APMAS2016), Maslak, Istanbul, Turkey, June 1-3, 2016

\title{
Mechanical Properties of Soda-Lime-Silica Glasses with Variable Peanut Shell Contents
}

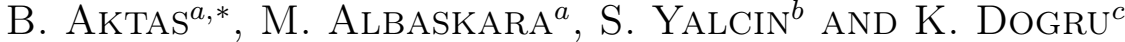 \\ ${ }^{a}$ Harran University, Engineering Faculty, Department of Mechanical Engineering, 63300, Sanliurfa, Turkey \\ ${ }^{b}$ Harran University, Arts and Sciences Faculty, Department of Physics, 63300, Sanliurfa, Turkey \\ ${ }^{c}$ Harran University, Institute of Natural and Applied Sciences, 63300, Sanliurfa, Turkey
}

\begin{abstract}
In this study, the mechanical properties of soda-lime-silica glasses were investigated by using micro-Vickers indentation tests, while varying the amount of peanut shell powder added to the glass. Peanut shell powder was obtained by grinding peanut shells. It was added as a dopant to glass at $0.5-5 \mathrm{wt} \%$, with the final glass samples being produced by melting. Peanut shell addition had led to a decreased density of the soda-lime-silica glasses, and X-ray diffraction data of the peanut shell-doped soda-lime-silica glasses indicated the presence of two crystalline phases, namely, anorthite $\left(\mathrm{Na}_{0.45}\left(\mathrm{Ca}_{0.55} \mathrm{Al}_{1.55} \mathrm{Si}_{2.45} \mathrm{O}_{8}\right)\right.$ and magnesium aluminum silicate $\left(\mathrm{MgAl}_{2} \mathrm{Si}_{3} \mathrm{O}_{10}\right)_{6}$. The Young's modulus values of the soda-lime-silica glasses increased along with the amount of peanut shell powder added. Peanut shell addition to the soda-lime-silica glasses has also caused increased hardness. Fracture toughness values of these glass samples were in the range of $0.91-1.04 \mathrm{MPa} \mathrm{m}^{1 / 2}$. Thus, the mechanical properties (e.g. $E$ and $K_{\mathrm{C}}$ ) of soda-lime-silica glasses could be enhanced through peanut shell addition.
\end{abstract}

DOI: 10.12693/APhysPolA.131.511

PACS/topics: 81.05.Kf, 81.70.--q, 64.70.P

\section{Introduction}

Glass is one of the most brittle materials in use today, and the catastrophic mechanical failure that it can experience is often attributed to this high brittleness. Glass has an equilibrium structure, which is obtained when a liquid is cooled down in such a way, that upon passing the melting temperature or liquidus temperature $T$, "freezing" occurs instead of crystallization [1]. Many scientists have classified the role of cations in glass structures into three groups based on coordination number [2], field strength [3], or electronegativity [4]. The first group of cations has coordination numbers of 3 or 4 , as well as high electronegativity values. These cations act as networkformers and produce reliable glasses ( $\mathrm{Si}, \mathrm{B}, \mathrm{P}, \mathrm{Ge}, \mathrm{As}, \mathrm{Be}$ with $\mathrm{F}$, etc.). The second group of cations (intermediate cations) behaves as either network formers or modifiers; they can either reinforce the network (coordination number 4 ) or loosen the network (coordination number 6-8). Such intermediate cations cannot form glasses by themselves. The last group (glass modifiers) has low electronegativity, and coordination numbers generally $>6$. These cations (including $\mathrm{Na}, \mathrm{K}, \mathrm{Ca}$, and $\mathrm{Ba}$ ) act to modify the network structure.

Soda-lime-silicate glass (SLS) is one of the most commonly used glass compositions and can be found in a wide range of glass products. For example, so-called solar control glass is a type of SLS glass containing around $1 \mathrm{wt} . \%$ of iron, which is used in automotive windows [5]. Iron is added to absorb ultraviolet (UV) and infrared

* corresponding author; e-mail: baktas@harran.edu.tr
(IR) solar radiation to reduce the harmful effects of UV rays and vehicle thermal load, to increase passenger comfort [6]. SLS glasses have also been investigated to determine whether their mechanical properties (such as elastic modulus and fracture toughness) could be used to predict glass hardness. The effect of alkaline earth oxide additives on the mechanical properties of SLS glasses were previously examined using micro-Vickers and four-point bend testing. The mechanical properties of SLS glasses with $\mathrm{MgO}-\mathrm{SiO}_{2}$ and $\mathrm{CaO}-\mathrm{SiO}_{2}$ deteriorate owing to the increased network depolymerization in their structures [7]. The objective of the present study, therefore, was to investigate the effects of alkali oxide powders (peanut shell powder) on the mechanical properties of SLS glasses, determined by using micro-Vickers hardness tester and an ultrasonic flaw detector.

\section{Materials and procedure}

In this study, SLS powders were used as the matrix material, and peanut shell (PS) powders were used as additives. SLS and PS powders were prepared by grinding from SLS glasses (Trakya Glass Industry Co.) and PS waste, respectively. The chemical compositions of the powders used in the study are given in Table I.

TABLE I

Chemical composition of powders used in the study.

\begin{tabular}{c|c|c|c|c|c|c|c|c|c}
\hline \hline & \multicolumn{7}{|c}{ Powder } & \multicolumn{10}{c}{$\%$} \\
\cline { 2 - 9 } & $\mathrm{SiO}_{2}$ & $\mathrm{Al}_{2} \mathrm{O}_{3}$ & $\mathrm{CaO}$ & $\mathrm{K}_{2} \mathrm{O}$ & $\mathrm{Fe}_{2} \mathrm{O}_{3}$ & $\mathrm{MgO}$ & $\mathrm{MnO}_{2}$ & $\mathrm{Na}_{2} \mathrm{O}$ & $\mathrm{TiO}_{2}$ \\
\hline $\mathrm{SLS}$ & 71.5 & 1.5 & 9.5 & - & 0.024 & 2 & - & 15.5 & 0.08 \\
PS & 0.789 & 4.812 & 29.732 & 13.268 & 3.966 & 9.148 & 1.655 & 36.633 & -
\end{tabular}

SLS powders were doped via mechanical mixing with PS powder in amounts of $0.5,1,3$ and 5 wt.\%, performed in an attritor type mixer at $200 \mathrm{rpm}$ for $1 \mathrm{~h}$. 
The powders were then pressed at $250 \mathrm{MPa}$ in a singleaxis die with a radius of $32 \mathrm{~mm}$, producing pellets $32 \mathrm{~mm}$ in diameter and $6 \mathrm{~mm}$ thick. Glass samples, undoped (as control) and PS-doped SLS glasses, were prepared via melting using a graphite mold, from pressed pellets at $1300^{\circ} \mathrm{C}$ for $2 \mathrm{~h}$ to ensure homogeneity and then the molten glass was quenched in the graphite mold. After quenching, samples were formed in a square graphite mold at $1100^{\circ} \mathrm{C}$. The formed samples were annealed at $500{ }^{\circ} \mathrm{C}$ for $3 \mathrm{~h}$ and then cooled slowly to room temperature inside the furnace. The surfaces of the samples were successively ground using 120, 240, 400, 600, 800 and $1200 \mathrm{SiC}$ grinding papers, and finally polished using 6,3 and $1 \mu \mathrm{m}$ diamond suspensions, for investigation of their microstructural and mechanical properties. X-ray diffraction (XRD) measurements of the samples were performed to identify the crystalline phases present, using XRD Rigaku D max 2000 with $\mathrm{CuK}_{\alpha}$ radiation, using "Jade" software, between $3^{\circ}$ and $60^{\circ}$ with a $3 \%$ s scan speed. The density of the samples was determined using the Archimedes principle with distilled water. Elastic modulus of the produced glasses was obtained by measuring the longitudinal and transverse ultrasonic wave velocities using Starmans Dio 1000 SFE. Both, hardness and fracture toughness of the specimens were determined using micro-Vickers hardness tester, using a load of $500 \mathrm{gf}$ and duration of $10 \mathrm{~s}$. Hardness values were calculated using the following equation:

$$
H_{\mathrm{V}}=\frac{1854 P}{d^{2}},
$$

where $P$ is the applied load $(\mathrm{kg})$ and $d$ is the mean value of the diagonal length $(\mathrm{mm})$. Fracture toughness values were calculated by measuring the lengths of the cracks that formed at the edges of the track resulted from the hardness tests. Cracks were measured immediately after applying the load to the specimen so that they would not be affected by environmental factors. Fracture toughness was calculated using the "half-penny-crack" formula proposed by Anstis et al. [8]:

$$
K_{\mathrm{IC}}=0.016\left(\frac{E}{H_{\mathrm{V}}}\right)^{\frac{1}{2}}\left(\frac{P}{C^{\frac{3}{2}}}\right),
$$

where $E$ is Young's modulus, $H_{\mathrm{V}}$ is the Vickers hardness, $P$ is the applied load, and $C$ is the crack length. Brittleness values $B$ were calculated from the measured $H_{\mathrm{V}}$ and $K_{\mathrm{IC}}$ values using

$$
B=\frac{H_{\mathrm{V}}}{K_{\mathrm{IC}}} \text {. }
$$

\section{Results and discussion}

XRD patterns of the glass samples doped with $(0.5,1$, 3 and 5 wt.\%) PS are shown in Fig. 1. These results show the presence of two crystalline phases, namely anorthite $\left(\mathrm{Na}_{0.45}\left(\mathrm{Ca}_{0.55} \mathrm{Al}_{1.55} \mathrm{Si}_{2.45} \mathrm{O}_{8}\right)\right.$; Pdf Number 01-078-0432) and magnesium aluminum silicate $\left(\mathrm{MgAl}_{2} \mathrm{Si}_{3} \mathrm{O}_{10}\right)_{6} ; \mathrm{Pdf}$ Number 01-073-2337). These spectra show amorphous bands at around $2 \theta=5^{\circ}$ and $2 \theta=25^{\circ}$, indicating the presence of a glassy phase [9]. Additionally, the dif- fraction peaks of PS-doped glasses are wider than those in pure soda lime glasses.

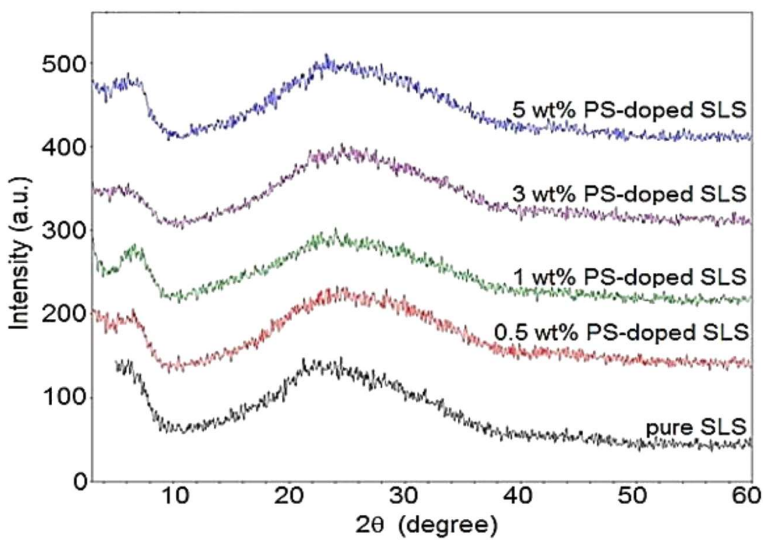

Fig. 1. X-ray diffraction patterns of SLS glass samples doped with PS powder.

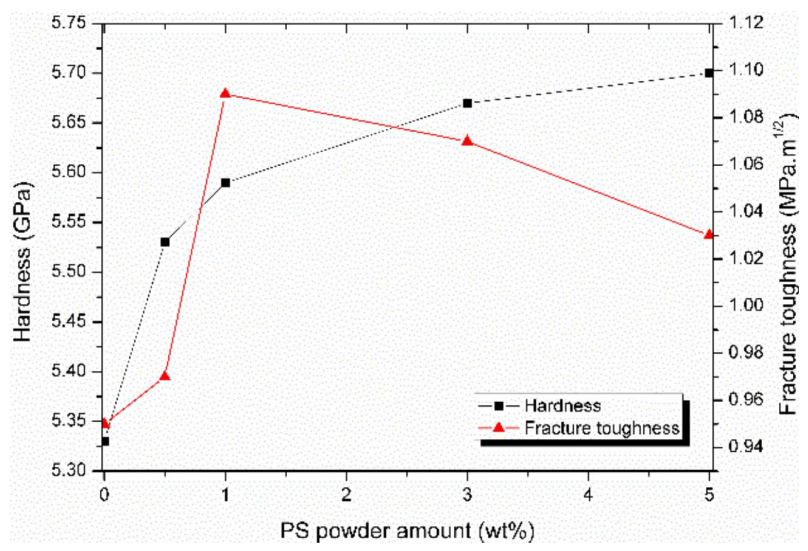

Fig. 2. Effects of PS contents on the hardness and the fracture toughness of SLS glass.

Figure 2 shows the effect of PS content on the hardness and fracture toughness of SLS glass. The hardness and fracture toughness of SLS glass samples increased with increased PS content, owing to the presence of $\mathrm{CaO}$ in the PS. When $\mathrm{CaO}$ is added to silica glass, it acts as a stabilizer, and increases the polymerization degree in the glass network by strengthening of the Si-O-Si bonds present in the glass. Si-O-Si covalent bonds constitute stronger bonds with $\mathrm{CaO}$ in silicate glasses, and these stronger bonds present in the doped samples increase the hardness and fracture toughness.

Table II summarizes the physical and mechanical properties of the glass samples. PS doping in SLS glasses causes decreased density in all samples. The reduced density of the doped SLS glasses is caused by $\mathrm{Na}_{2} \mathrm{O}$ with low density, present within the PS powders (Table I). Hardness evaluation for these glasses is very complex, as it is affected by a number of different factors, such as degree of polymerization, bond strength and fictive temperature [10]. Elastic modulus, hardness and fracture toughness of SLS glasses have increased as more PS was 
added. This improvement of these mechanical properties in the doped samples is likely due to increased network conductivity by means of polymerization, through strengthening of the $\mathrm{Si}-\mathrm{O}-\mathrm{Si}$ bonds as was discussed above.

TABLE II

Physical and mechanical properties of pure and PS-doped SLS glasses.

\begin{tabular}{c|c|c|c|c|c}
\hline \hline Glass structure & $\begin{array}{c}E \\
{[\mathrm{GPa}]}\end{array}$ & $\begin{array}{c}\rho \\
{\left[\mathrm{kg} / \mathrm{m}^{3}\right]}\end{array}$ & $\begin{array}{c}H_{\mathrm{V}} \\
{[\mathrm{GPa}]}\end{array}$ & $\begin{array}{c}K_{\mathrm{IC}} \\
{\left[\mathrm{MPa} \mathrm{m}^{1 / 2}\right]}\end{array}$ & $\begin{array}{c}\text { Brittleness } B \\
{\left[\mu \mathrm{m}^{-1 / 2}\right]}\end{array}$ \\
\hline Pure SLS & 71.94 & 2513 & $5.33 \pm 0.12$ & 0.91 & 5.61 \\
0.5 wt.\% PS-doped SLS & 72.18 & 2512 & $5.53 \pm 0.12$ & 0.93 & 5.70 \\
1 wt.\% PS-doped SLS & 72.43 & 2511 & $5.59 \pm 0.21$ & 1.04 & 5.13 \\
3 wt.\% PS-doped SLS & 72.79 & 2508 & $5.67 \pm 0.24$ & 1.02 & 5.30 \\
5 wt.\% PS-doped SLS & 74.33 & 2505 & $5.70 \pm 0.27$ & 0.98 & 5.53
\end{tabular}

Figure 3 shows the Vickers indentation imprints for undoped and PS-doped SLS samples. All glass samples exhibited cracking at the four corners of the indent at $500 \mathrm{gf}$ load for $10 \mathrm{~s}$ in air. Median and radial cracks were generally observed in the undoped and PS-doped samples (Fig. 3a-e).

\section{Conclusions}

The mechanical properties of SLS glasses were improved through PS doping at different contents. As PS addition into glasses increases the degree of polymerization within the glass network is strengthening the $\mathrm{Si}-\mathrm{O}-\mathrm{Si}$ bonds. The elastic modulus, hardness and fracture toughness of SLS glasses could be improved through the addition of PS. Brittleness has also decreased as PS was added. Furthermore, indentation imprints data demonstrate that the glass cracking resistance increases with the increasing amounts of PS.

\section{Acknowledgments}

This work was supported in part by HUBAK (the scientific research projects commission of Harran University, Sanliurfa, Turkey) under project numbers 14170 and K16046. The author is grateful to this commission for financial support.

\section{References}

[1] G. Tammann, The States of Aggregation, D. Van Nostrand, New York 1925, p. 297.

[2] W.H. Zachariasen, J. Am. Chem. Soc. 54, 3841 (1932).

[3] W. Vogel, Chemistry of Glass, The American Ceramic Society, Columbus, OH 1985, p. 113.

[4] J.E. Stanworth, Physical Properties of Glass, Oxford University Press, London 1950, p. 39.

[5] T. Uchino, K. Nakaguchi, Y. Nagashima, T. Kondo, J. Non-Cryst. Solids 261, 72 (2000).

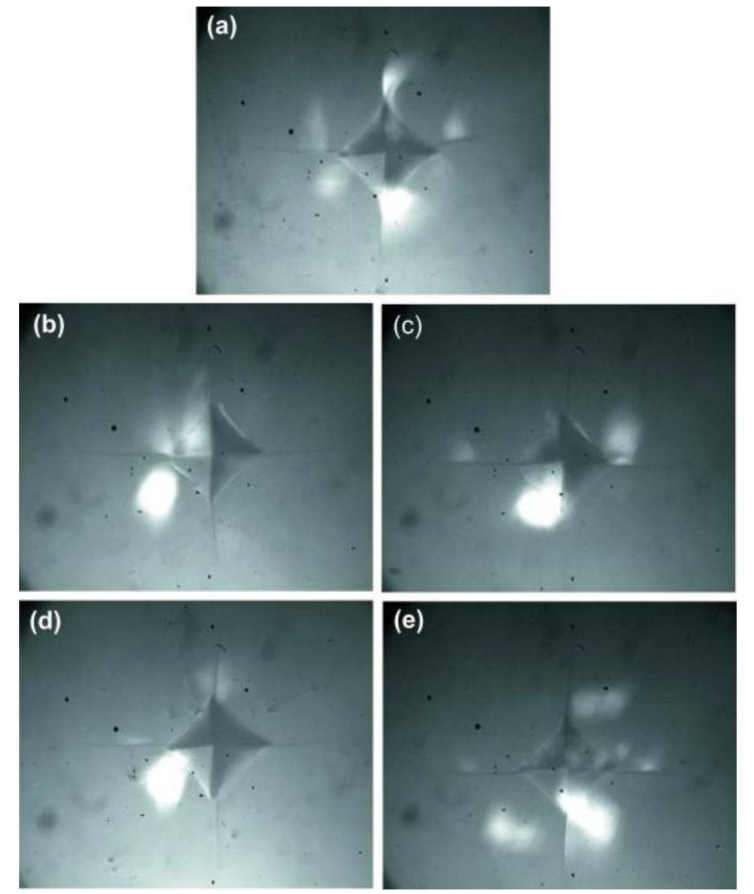

Fig. 3. Micrographs of Vickers indentations with different crack lengths of (a) undoped, (b) 0.5, (c) 1, (d) 3 and (e) 5 wt.\% PS-doped SLS glasses.

[6] L. Pilon, F. Janos, R. Kitamura, J. Am. Ceram. Soc. 97, 442 (2014).

[7] E. Kilinc, R.J. Hand, J. Non-Cryst. Solids 429, 190 (2015).

[8] G.R. Anstis, P. Chantikul, B.R. Lawn, D.B. Marshall, J. Am. Ceram. Soc. 64, 533 (1981).

[9] S. Lopez-Esteban, J.F. Bartolome, L.A. Diaz, L. Esteban-Tejeda, C. Prado, R. Lopez-Piriz, R. Torrecillas, J.S. Moya, J. Mech. Behav. Biomed. Mater. 34, 302 (2014).

[10] M.M. Smedskjaer, M. Jensen, Y. Yue, J. Non-Cryst. Solids 356, 893 (2010). 